\title{
Mitochondrial antioxidant defence in radio-resistant Lepidopteran insect cells
}

\author{
Shubhankar Suman ${ }^{1,2}$, Rakesh Kumar Seth ${ }^{2}$, Sudhir Chandna ${ }^{1 *}$ \\ ${ }^{\mathbf{1}}$ Natural Radiation Response Mechanisms (NRRM) Group, Division of Radiation Biosciences, Institute of Nuclear Medicine \& Allied \\ Sciences, Brig S K Mazumdar Road, Delhi-110054, India; ${ }^{2}$ Department of Zoology, University of Delhi, Delhi-110007, India; Sudhir \\ Chandna * - Email: sudhirchandna@yahoo.com; *Corresponding Author
}

Received April 01, 2009; Revised May 26, 2009; Accepted June 13, 2009; Published August 18, 2009

\begin{abstract}
:
Cells isolated from Lepidopteran insects (butterfly and moths) display very high radioresistance as compared to mammals and other insect species. Since free radical induced mitochondrial damage under stress conditions is very crucial for cellular fate determination, antioxidant system is the major protective modality required to minimize stress-induced damage and to modulate cellular sensitivity. In this study, we predict the mitochondrial localization potential and co-existence of important antioxidant enzymes in insect cells and compare with other radiosensitive (mammals, Dipteran insects) and radioresistant (nematodes) species. Our study clearly demonstrates the inter-species variation in then localization potential of various antioxidant enzymes. A higher mitochondrial localization potential as a function of mitoprot score was evident for all important antioxidant enzymes in the lepidopteran insect Bombyx mori (Mn-SOD, 0.694; GPx, 0.862; TRPx, 0.997; TR, 0.9), besides an unusual mitochondrial localization prediction for catalase $(0.453)$. We further found coexistence of glutathione and thioredoxin system in the mitochondria of lepidopteran insects as also reported in various plant species. On the basis of above observations, we hypothesize that a strong mitochondrial antioxidant enzyme system including the unusual coexistence of catalase, glutathione and thioredoxin system may help minimize the free radical mediated damage to mitochondria and can contribute to the intrinsic radioresistance of lepidopteran insects.
\end{abstract}

Keywords: Mitochondrial DNA (mtDNA), Manganese-superoxide dismutase (Mn-SOD), Glutathione peroxidase (Gpx), Gluthathione reductase (GR), Glutathione S-transferase (GST), Thioredoxin peroxidase (TRPx) and Thioredoxin reductase (TR)

\section{Background:}

Mitochondrion is a semi-autonomous organelle involved in several vital cellular processes including energy production, fatty acid metabolism, calcium homeostasis and regulation of cell death. This is the only organelle that contains extranuclear DNA in animal cells. The mitochondrial genome (mtDNA) encodes 13 polypeptides involved in oxidative phosphorylation as well as 22 tRNA and two rRNA species. The mitochondrial structure is highly susceptible to oxidative stress as shown by reports on lipid peroxidation [1], protein oxidation [2] and genomic alterations in mitochondria. Higher damage susceptibility of mtDNA than nuclear DNA can be explained on the basis of (a) absence of introns, (b) lack of histones and other shielding proteins, (c) presence in the close vicinity of electron transport chain, a major source of cellular ROS, and (d) lack of proactive DNA repair system in mitochondria [3]. Ionizing radiation can increase ROS level through hydrolysis of water as well as through damage to ETC proteins and/or mtDNA. Ionizing radiation induced mtDNA damage and mitochondrial dysfunction have also been implicated in chromosomal instability through perpetuation of oxidative stress in irradiated cells [4]. In an experiment with targeted cytoplasmic irradiation, induction of mutation in nuclear DNA has been observed, which further suggests the importance of mitochondrial dysfunction in radiation-induced cytotoxicity [5]. Mitochondrial DNA damage is also known to initiate apoptosis as it leads to further increase in leakage of electrons from ETC and generation of superoxide radicals followed by activation of mitochondrial membrane permeabilization and cytochrome-c release [6]. Several antioxidant enzymes (SOD (Superoxide dismutase), Catalase, Peroxidases, GST (Glutathione S-transferase)) and a network of low molecular weight antioxidants (ascorbate, glutathione, tocopherol and phenolic compounds) are known to protect the cells from radiation-induced ROS. SOD catalyzes the dismutation of superoxide radical to hydrogen peroxide and is present in all aerobic organisms. There are two types of SOD enzymes present in mammalian cells, viz., $\mathrm{Cu}-\mathrm{Zn}$ SOD (cytoplasmic/ nuclear) and Mn-SOD (mitochondrial). Hydrogen peroxide generated after SOD activity is further converted into water by catalase and peroxidases. Peroxidases constitute a coenzyme based enzymatic system that utilizes low molecular weight antioxidants such as Glutathione (GSH), ascorbate (AA) and thioredoxin (TRx) to convert hydrogen peroxide into water, e.g., glutathione-, thioredoxin- and ascorbate-peroxidase,

ISSN 0973-2063 (online) 0973-8894 (print)

Bioinformation 4(1): 19-23 (2009) respectively. Each of these three redox systems includes one peroxidase for removal of hydrogen peroxide as well as a reductase to maintain the redox status. Reduced forms of these are glutathione, ascorbic acid and reduced thioredoxin, which are well known antioxidants and can scavenge radicals such as hydroxyl radicals. The glutathione system includes glutathione (GSH), glutathione reductase (GR), glutathione peroxidase (GPx) and glutathione S transferase (GST). This system is found in animals, plants and micro-organisms and is required for removal of hydrogen peroxide and organic hydroperoxides such as lipid hydroperoxides [7]. The thioredoxin system contains the $12 \mathrm{kDa}$ protein thioredoxin and its regulatory enzymes thioredoxin peroxidase (TRPx) and thioredoxin reductase (TR). Proteins related to thioredoxin system are present in most of the sequenced organisms. The active site of thioredoxin consists of two cysteines, as a CXXC conserved motif and can cycle between an active reduced di-thiol and oxidized disulfide form. In its active state, thioredoxin acts as a good reducing agent and ROS scavenger and maintains other proteins in the reduced form [8]. In addition to these two systems, ascorbate system has been reported in various plants and few insect species, which consist of its peroxidase and reductase enzymes. Ascorbate system has been considered as the stronger antioxidant defence in plant species. Among the naturally existing radioresistant organisms, insects are evolutionarily closest to mammalian system and yet display very high radioresistance. Lepidopteran insects and cells have comparable radioresistance as nematodes, which is roughly $>10$ times and 50-100 times more than Dipteran insects and mammals, respectively [9]. Studies so far have shown that reduced DNA damage, enhanced DNA repair, lack of chromosome breakage and resistance against radiation-induced apoptosis contribute to Lepidopteran radioresistance, besides other mechanisms [9-11]. Since oxidative stress involving mitochondria plays important role in radiation response and antioxidant enzymes are known to modulate cellular responses to radiation and other stress agents, the current study aims to analyze the presence of antioxidant enzymes in mitochondria of different species including mammals and stressresistant insects and nematodes. Our analysis clearly indicates higher Mitoprot scores indicating preferred availability/ targeting of antioxidant enzymes in the mitochondria of insects and nematodes than in relatively radiosensitive mammalian mitochondria. This may favour enhanced antioxidant capacity of Lepidopteran insect cells as well as nematodes, and may contribute to reduced radiation-induced 19 
mitochondrial dysfunction and resistance against radiation-induced apoptosis observed in Lepidopteran Sf9 cells [9].

\section{Methodology:}

Sequence retrieval and data set:

Full length protein sequences for all the antioxidant enzymes including manganese-superoxide dismutase, catalase, glutathione peroxidase, gluthathione reductase, glutathione S-transferase, thioredoxin peroxidase and thioredoxin reductase were retrieved from Genebank using protein and species name or accession number as keywords. Representative protein sequences for all antioxidant enzymes derived from nematode, insects and mammals were considered for analysis. Accession numbers for all the sequences used for analysis are listed in table 1. Import of many nuclear encoded mitochondrial proteins is mediated by mitochondrial targeting signal (MTS). This N-terminal presequence is usually enriched in positive, hydrophobic and hydroxylated amino acids and devoid of acidic amino acids. Since mitochondrial localization is influenced by $\mathrm{N}$-terminal sequence of the protein, all retrieved protein sequences were manually checked for intact $\mathrm{N}$-terminus and sequences starting from methionine as first amino acid were picked for further analysis.

Data analysis:

Extent of mitochondrial localization for all retrieved sequences was predicted using Mitoprot server (ftp://ftp.biologic.ens.fr/pub/molbio, institute of human genetics, Helmholtz center munich). Mitoprot scores and the other information given by mitoprot server such as the presence of target sequence, cleavage site in the target sequence, net charge on protein sequence, number of basic and acidic residues, hydrophobic moment $(\mu \mathrm{H} \delta)$ and Hydrophobicity maxima (Hmax) in target peptide were also considered for the analysis.

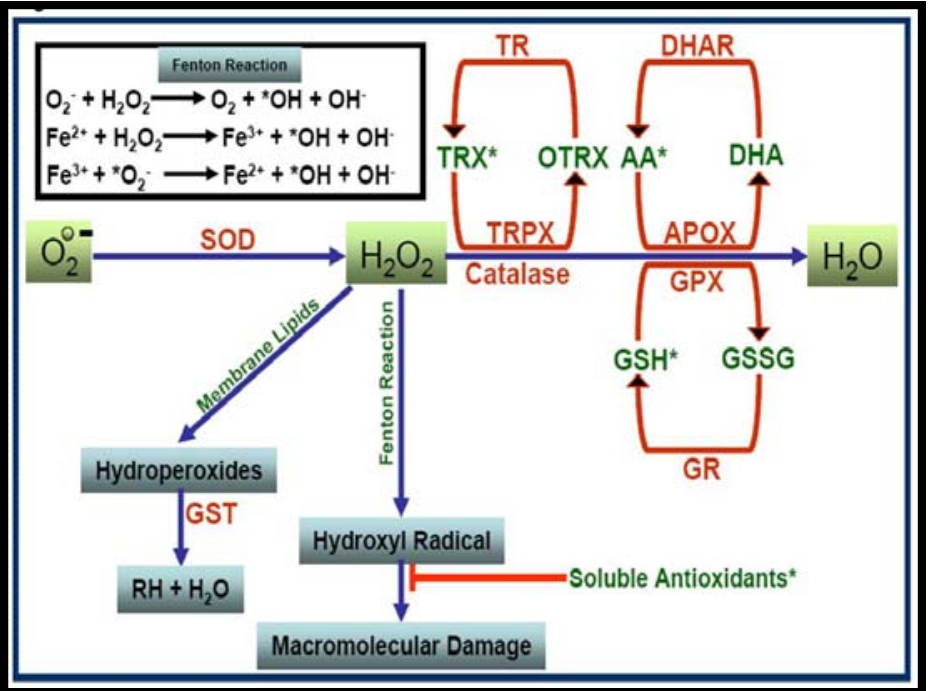

Figure 1: Interaction between free radicals and the antioxidant defence system present in living organisms. Superoxide radical $\left(\mathrm{O}_{2}{ }^{-}\right)$is converted into hydrogen peroxide $\left(\mathrm{H}_{2} \mathrm{O}_{2}\right)$ by superoxide dismutase (SOD) enzyme. $\mathrm{H}_{2} \mathrm{O}_{2}$ may further react with membrane lipids, undergo Fenton chain reaction and can be converted into nontoxic $\mathrm{H}_{2} \mathrm{O}$ by various antioxidant system including glutathione (GSH: reduced glutathione, GSSG: oxidized glutathione, Gpx: glutathione peroxidase, GR: glutathione reductase), ascorbate (AA: reduced ascorbate, DHA: dehydroascorbate, Apox: ascorbate peroxidase, DHAR: dehydro-ascorbate reducatse), thioredoxin (TR: thioredoxin reductase, Trpx: thioredoxin peroxidase, Trx: reduced thioredoxin, OTrx: oxidized thioredoxin) and catalase. Lipid hydroperoxides and hydroxyl radicals generated by $\mathrm{H}_{2} \mathrm{O}_{2}$ are further scavenged by Glutahione S-transterase and soluble antioxidants.

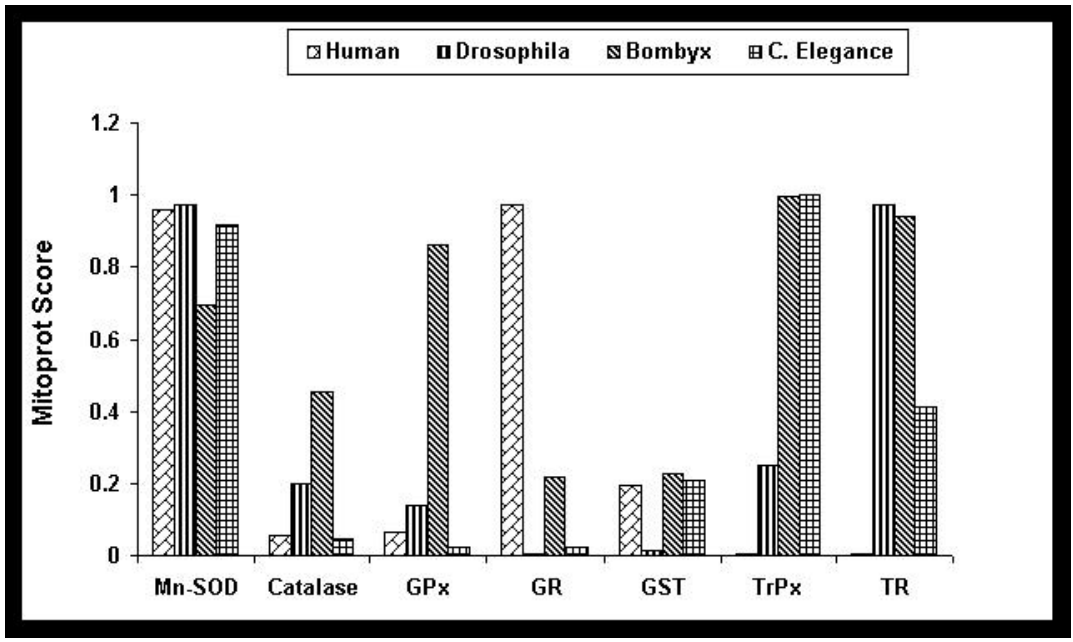

Figure 2: Mitoprot score of various antioxidant enzymes from Mammalia (Homo sapiens), Insecta (Drosophila melanogaster and Bombyx mori) and Nematoda (C. elegans).

ISSN 0973-2063 (online) 0973-8894 (print) 
Discussion:

Mitochondrial localization of antioxidant enzymes

Majority of mitochondrial proteins are encoded in the nucleus, synthesized in the cytosol and are imported back into mitochondria with the help of mitochondrial targeting signal (MTS). This Nterminal MTS is usually enriched in positive hydrophobic amino acids and is devoid of acidic amino acids. The MTS has a tendency to form an amphiphylic $\alpha$-helix, which presents a positively charged hydrophobic surface. The hydrophobic moment $(\mathrm{mHd}$; used as a measure of the helical amphiphilicity), asymmetric distribution of hydrophobic side chains and the maximal hydrophobicity (Hmax) of hydrophobic face of helical structure are also very important for mitochondrial targeting of a protein [12-14]. All these properties important for mitochondrial localization of antioxidant enzymes are described in table-1. Non-mitochondrial proteins are known to have generally lower hydrophobic moment and lower number of positively charged amino acids. Listed observations in Table-1 clearly suggest that all representative species from nematodes, insects and mammals have different set of antioxidant enzymes with predicted mitochondrial localization. Mn-SOD was the only enzyme found to have higher $\mathrm{mHd}$, Hmax, mitoprot score, number of basic residues and higher net charge in all the species studied and seems to be exclusively mitochondrial in insect, nematode and mammals. Although no signal peptide and signal cleavage site was predicted in nematode Mn-SOD, other features were found similar to other MnSOD orthologues while showing higher hydrophobicity, thereby enhancing its mitoprot score. Mitochondrial localization of catalase, which is usually abundant in cytosol, has been known to provide resistance against ionizing radiation and oxidative stress [15]. This unusual intracellular distribution of catalase has been seen in another lepidopteran species Trichoplusia ni, with $47 \%$ catalase activity from mitochondrial fraction [16]. In line with the observation in Trichoplusia ni, catalase mitochondrial import prediction of Lepidopteran insects (Bombyx mori) was significantly higher than the relatively radiosensitive Dipteran insects (Drosophila) and mammalian species. In addition to catalase, thioredoxin system enzymes predict higher localization potential in insects and nematodes (Figure 2) as compared to mammals, which may contribute to their stress resistance.

\section{Mitochondrial thioredoxin and glutathione system}

The thioredoxin and glutathione enzyme systems regulate cellular redox potential and the maintenance of reduced (thiol-rich) intracellular state, which helps in damage minimization. Parallel functioning of thioredoxin and glutathione system provides considerable protection against oxidative stress [8]. Our analysis of mitochondrial localization of these antioxidant enzymes clearly suggests that all the components of thioredoxin and glutathione system are present in the mitochondria of Lepidopteran system and can scavenge reactive oxygen species (ROS). Coexistence of the ascorbate and glutathione system has been reported in some plant species [17] and our prediction also suggests the analogous antioxidant enzyme coupling in lepidopteran insects. Other species analyzed have strong presence of only either thioredoxin system (Diptera, Nematoda) or glutathione system (Mammalia). Factors responsible for mitochondrial localization of thioredoxin and glutathione system enzymes listed in table-1 suggest that the protein sequences of these enzymes are responsible for their differential distribution in mammalian and invertebrate system along with the presence of mitochondrial localization signal. In addition to these factors, active ascorbate system has been reported in Lepidopteran insects $[18,19]$. Enzymes associated with the ascorbate system such as ascorbate peroxidase and dihydroascorbate reductase have been found active in many different species of insect larvae, suggesting its wide presence among insects [20-22]. Altogether, the strong presence of thioredoxin-glutathione coupled system and catalase in the mitochondria of lepidopteran insect along with strong ascorbate peroxidase activity may help reduce mitochondrial damage and contribute in the oxidative stress resistance.

\section{Conclusion:}

The present computational analysis of antioxidant enzymes from different organisms demonstrates that strong insect mitochondrial antioxidant system might be a function of the preferred localization of certain antioxidant enzymes in mitochondria. Specifically, a strong glutathione and thioredoxin system (as indicated by the mitoprot score) in lepidopteran insects as well as active ascorbate system [18, 19] can effectively modulate stress sensitivity. We hypothesize that these features can significantly reduce the risk of damage to insect mtDNA, proteins and cell membranes by radiation and other stressors, and could significantly contribute towards the radio-resistance of lepidopteran cells.

References:

[1] AM Hruszkewyez, Mutat. Res. 275:243 (1992) [PMID: 1383766]

[2] ER Stadtman, Science 257:1220 (1992) [PMID: 1355616]

[3] C Richter, Mutat. Res. 275: 249 (1992) [PMID: 1383767]

[4] GJ Kim et al., Mutagenesis 21:361 (2006) [PMID: 17065161]

[5] LJ Wu et al., Proc. Natl. Acad. Sci. USA 96:4959 (1999) [PMID: 10220401]

[6] C Ricci et al., Am. J. Physiol. Cell. Physiol. 294:413 (2008) [PMID: 18077603]

[7] A Holmgren et al., Biochem. Soc. Trans. 33:1375 (2005) [PMID: 16246122]

[8] H Zhang et al., Arch. Biochem. Biophys. 465:119 (2007) [PMID: 17548047]

[9] S Chandna et al., Int. J. Radiat. Biol. 80:301 (2004) [PMID 15204707]

[10] TM Koval, Proc. Natl. Acad. Sci. USA 80:4752 (1983) [PMID: 16593348]

[11] S Suman et al., Bioinformation 3:8 (2008) [PMID: 19052659]

[12] M Dinur-Mills et al., PLOS One 3:e2161 (2008) [PMID: 18478128]

[13] MG Calros, P Vincens, Eur. J. Biochem. 241:779 (1996) [PMID: 8944766]

[14] D Rapaport, EMBO reports 4:948 (2003) [PMID: 14528265]

[15] Y Arita et al., Am. J. Physiol. Lung Cell Mol. Physiol. 290:L978 (2006) [PMID: 16387755]

[16] S Ahmad et al., Free Radiac. Res. Commun. 4:403 (1988) [PMID: 3243504]

[17] O Chew et al., J. Biol. Chem. 278:46869 (2003) [PMID: 12954611]

[18] Y Wang et al., Free. Radic. Biol. Med. 30:1254 (2001) [PMID: 11368923]

[19] Y Wang et al., Free. Radic. Biol. Med. 31:1448 (2001) [PMID: 11728817]

[20] CB Summers, GW Felton, Biochim. Biophys. acta. 1156:235 (1993) [PMID: 8427882]

[21] GW Felton and C. B. Summers, Arch. Insect Biochem. Physiol. 29:187 (1995) [PMID: 7606043]

[22] N Krishnan, F Sehnal, Arch. Insect Biochem. Physiol. 63:1 (2006) [PMID: 16921519]

Edited by P. Kangueane

Citation: Suman et al, Bioinformation 4(1): 19-23 (2009)

License statement: This is an open-access article, which permits unrestricted use, distribution, and reproduction in any medium, for noncommercial purposes, provided the original author and source are credited. 


\section{Supplementary material}

Table 1: Biochemical features important for mitochondrial localization of antioxidant enzyme.

\begin{tabular}{|c|c|c|c|c|c|}
\hline Homo sapiens (Mammalia) & mHd/Hmax & Signal peptide & $\begin{array}{l}\text { Basic/acidic } \\
\text { residues }\end{array}$ & Net charge & Mitoprot Score \\
\hline Mn-SOD (NP_001019636.1) & $28.65 / 12.3$ & Present & $4 / 0$ & +2 & 0.9575 \\
\hline CAT (CAA27721.1) & $26.96 /-2.325$ & NP & $0 / 0$ & -2 & 0.0548 \\
\hline GPx (BAA00525.1) & $18.29 / 17.4$ & Present & $2 / 0$ & +2 & 0.2879 \\
\hline GR (NP_000628.2) & $50.24 / 16.8$ & Present & $10 / 3$ & +7 & 0.9718 \\
\hline GST (CAA48637.1) & $17.41 / 13.6$ & $\mathrm{NP}$ & $0 / 0$ & -4 & 0.1926 \\
\hline Trpx (NP_006397.1) & $19.64 / 8.74$ & NP & $0 / 0$ & -6 & 0.0057 \\
\hline TR (AAB35418.1) & $22.14 / 5.45$ & NP & $0 / 0$ & -5 & 0.0033 \\
\hline \multicolumn{6}{|c|}{ Drosophila melanogaster (Insecta, Diptera) } \\
\hline Mn-SOD (NP_476925.1) & $27.24 / 7.89$ & Present & $5 / 0$ & +1 & 0.9716 \\
\hline CAT (CAA36529.1) & $16.62 /-0.8$ & NP & $1 / 0$ & +4 & 0.1887 \\
\hline GPx (CAA48238.1) & $21.179 / 14.54$ & NP & $4 / 1$ & -14 & 0.1375 \\
\hline GR (AAB48441.1) & $4.833 / 8.423$ & NP & $0 / 0$ & 0 & 0.0040 \\
\hline GST (NP_725653.1) & $19.51 / 6.67$ & NP & $0 / 0$ & -18 & 0.0162 \\
\hline Trpx (NP_727689) & $14.689 / 12.4$ & NP & $2 / 0$ & -2 & 0.2565 \\
\hline TR (NP_727251.1) & $42.36 / 11.2$ & Present & $11 / 2$ & +3 & 0.9737 \\
\hline \multicolumn{6}{|c|}{ Bombyx mori (Insecta, Lepidoptera) } \\
\hline Mn-SOD (BAD51413.1) & $17.99 / 5.752$ & Present & $4 / 0$ & +3 & 0.694 \\
\hline CAT (NP_001036912.1) & $23.59 / 3.3$ & NP & $1 / 0$ & +2 & 0.4536 \\
\hline GPx (NP_001036999.1) & $30.207 / 18.91$ & Present & $4 / 0$ & +4 & 0.8620 \\
\hline GR (NP_001040197.1) & $28.74 / 18.3$ & NP & $5 / 0$ & +4 & 0.2166 \\
\hline GST (CAA07071.1) & $23.07 / 11.02$ & NP & $3 / 0$ & -7 & 0.2275 \\
\hline Trpx (NP_001040464.1) & $41.50 / 12.04$ & NP & $8 / 0$ & +2 & 0.9978 \\
\hline TR (ABF51426.1) & $35.33 / 13.08$ & Present & $4 / 0$ & 0 & 0.9417 \\
\hline \multicolumn{6}{|c|}{ Caenorhabditis elegans/*Brugia malayi (Nematoda) } \\
\hline Mn-SOD (BAA02363.1) & $34.84 / 16.5$ & NP & $4 / 0$ & +1 & 0.9176 \\
\hline CAT (CAA57665.1) & $26.81 /-2.825$ & NP & $0 / 0$ & +1 & 0.0457 \\
\hline GPx (Q95003.1) & $6.169 / 17.72$ & NP & $0 / 0$ & -11 & 0.0246 \\
\hline GR* (XP_001892443.1) & $24.06 / 7.47$ & NP & $0 / 0$ & +3 & 0.0239 \\
\hline GST (AAB65417.1) & $18.49 / 10.43$ & NP & $1 / 0$ & -3 & 0.2065 \\
\hline $\operatorname{Trpx}(\mathrm{Q} 21824)$ & $36.28 / 16.67$ & NP & $7 / 0$ & 0 & 0.9989 \\
\hline TR (AAD41826.1) & $36.87 / 8.36$ & Present & $1 / 0$ & -6 & 0.4118 \\
\hline
\end{tabular}

Table 1: Predicted N-terminal mitochondrial signal cleavage sites in antioxidant enzymes.

\begin{tabular}{ll}
\hline Enzyme & Predicted Signal Peptide Cleavage Site \\
\hline Mn-SOD (Human) & 21 MLSRAVCGTSRQLAPVLGYL \\
Mn-SOD (Drsophila) & $\mathbf{1 5}$ MFVARKISQTASLA \\
Mn-SOD (Bombyx) & $\mathbf{1 4}$ MLMSQRIGSLIRV \\
Mn-SOD (C. elegans) & Not Predicted \\
GPx (Human) & $\mathbf{1 3}$ MARLLQASCLLS \\
GPx (Drosophila) & Not Predicted \\
GPx (Bombyx) & $\mathbf{3 3}$ MTISRFVIAKLATPIIGNICLSRAQLSTVRM \\
GPx (C. elegans) & Not Predicted \\
GR (Human) & $\mathbf{8 4}$ MALLPRALSAGAGPSWRRAARAFRGFLLLLPEPALLTRALSRAMACRQEPQPQGPPPAAG \\
GR (Drosophila) & AVASYDYLVIGGGSGGLASARR \\
GR (Bombyx) & Not Predicted \\
GR (B. malayi) & Not Predicted \\
TrPx (Human) & Not Predicted \\
TrPx (Drosophila) & Not Predicted \\
TrPx (Bombyx) & Not Predicted \\
\end{tabular}




\begin{tabular}{ll} 
TrPx (C. elegans) & $\mathbf{4 1}$ MFSSAVRALCRTVPTVATRQLSTSRALLSLRPLGPKNTVP \\
TR (Human) & Not Predicted \\
TR (Drosophila) & $\mathbf{1 0 6}$ MNLCNSRFSVTFVRQCSTILTSPSAGIIQNRGSLTTKVPHWISSSLSCAHHTFQRTMNLTG \\
TR (Bombyx) & QRGSRDSTGATGGNAPAGSGAGAPPPFHPHCDRAAMYAQPVRK \\
TR (C. elegans) & Not MLSSLRTGVQGLWRI \\
\hline
\end{tabular}

Number of amino acids present in signal sequence is represented in bold numeric. 